\title{
A CADAVERIC STUDY: THE MORPHOMETRIC DIFFERENCES IN THE LEVATOR SCAPULAE MUSCLE
}

\author{
Jan H.T. Smit ${ }^{1}$, Matthew R.H. Todd ${ }^{2}$ \\ ${ }^{1}$ Department of Anatomy, School of Medicine, Faculty of Health Sciences, University of Namibia, \\ Windhoek, Namibia. 9000. jsmit@unam.na \\ ${ }^{2}$ School of Medicine, Queens University of Belfast, Belfast, Northern Ireland. \\ Correspondence to: Prof. Jan H.T. Smit, PO Box 87188, Eros, Windhoek, Namibia. 9000. \\ Tel:+264 61 2065099; jsmit@unam.na
}

\begin{abstract}
Although the levator scapulae are surrounded in the deep cervical fascia, it can be separated into a number of muscle slips at the proximal attachment. Originally the muscle was described as having 3 muscle slips at its origin. More recent texts have now adopted 4 slips of origin. Each slip coming from a different cervical vertebra. Levator scapulae is important with myofascial pain syndrome which is one of the leading causes of neck and shoulder pain. Surgically it can also be used to overcome trapezius paralysis. Anatomical variations of the levator scapulae are important and therefore clinically relevant. In this dissection study, we have investigated the morphometric differences in 46 levator scapulae muscles from 23 cadavers. Measurements of the proximal- and distal attachments and the total length of the muscles were taken. Between 3 and 6 muscle slips were reported at the proximal attachment. Differences were also observed between sides. The first report of a levator scapula muscle with 6 muscle slips at the proximal attachment, is described in this study.
\end{abstract}

Key words: Origin (proximal attachment); insertion (distal attachment); muscle slips.

\section{INTRODUCTION}

In 1867 Wood was the first to describe 4 muscle slips of levator scapula at the proximal attachment as the norm. Before his report, 3 muscle slips were always accepted as norm at the origin (Wood, 1867). During a later study done in Japan in 1964, levator scapulae with 3-5 muscle slips were reported in their investigation (Mori, 1964). Over time many other accessory attachments of the levator scapula have also been reported. Common variations include; accessary attachment to the occipital bone, mastoid process, ribs one and two, with the trapezius muscle, scalene muscles or even with the serratus muscles. The extent to which the slips separate and reunite also varied greatly (Standring, 2005).

In 2006 an accessory slip of levator scapulae muscle was reported to insert onto the ligamentum nuchae, rhomboid major tendon and also serratus posterior superior muscle (Loukas et al., 2006). A deviant muscle slip, originating from the upper cervical vertebrae and attaching to the clavicle was also described. This, however, is now considered as a separate muscle and named levator clavicular which is present in $2-3 \%$ of the population (Rubinstein et al., 1999).

The levator scapulae are now believed to be the leading cause of discomfort in patients with chronic tension-type neck and shoulder pain and a link between anatomical variants of the muscle and increased risk of developing pain has been speculated (Menachem et al., 1993). In addition to this, the position of levator scapula within the floor of the posterior triangle of the neck, make it clinically important for interventional procedures which requires brachial plexus nerve blocks. Consequently, research outlining the anatomical variants of levator scapulae would be of interest to several clinical applications. Limited research and insufficient reports are available on these variations of the levator scapulae. With this study we look at the morphometric differences 
of the levator scapulae muscles in the cadaver population at the Anatomy department of Queens University in Belfast.

\section{MATERIALS \& METHODS}

Ethical clearance for the study was obtained from the Ethical committee of the School of Medicine at Queens University in Belfast. All the specimens used during the research were obtained through the "Body Bequest Scheme" of the Anatomy Department at Queens University. Each cadaver was embalmed via the right common carotid artery with a mechanical pump infusion over a period of twelve hours. To investigate the levator scapulae muscles, a complicated dissection procedure was developed. The aim was to fully expose the muscle along its entire length. In order to achieve this, the cadaver had to be dissected in the prone- and supine positions to see the origin and the insertion properly. Once this was done, measurements were taken, and descriptions done and documented.

For adequate exposure of the levator scapulae, the dissection was done in two stages. The posterior approach helped to define the insertion of the muscle onto the scapula. The anterolateral approach was to expose the origin of the muscle from the cervical vertebrae. The technique used for dissection was primarily blunt dissection with fingers and forceps. A scalpel was only used for making skin incisions and the scissors for blunt dissection and reflecting of neighboring muscles.

The posterior approach began with the cadaver placed in the prone position. The neck was flexed, and the thorax was lifted by a head support block. Using a disposable surgical scalpel, a midline skin incision was made, starting at the external occipital protuberance, and ending at an imaginary line between the inferior borders of the right and left scapulae. Both these landmarks can easily be palpated beneath the skin. Two other skin incisions were made perpendicular to the midline incision in order to reflect the skin.
The skin and superficial fascia were reflected laterally to expose the back muscles. Trapezius was the first major muscle to be seen. Trapezius was cleared of fascia and fatty tissue, before reflecting the muscle laterally towards its insertion.

The surface of the rhomboids was cleaned to establish the inferior end of the levator scapula insertion. The distal attachment of levator scapula onto the medial border of the scapula and the direction of the muscle towards the cervical vertebrae, was now exposed. The levator scapulae are surrounded by large amounts of loose connective tissue and dense irregular connective tissue. These were cleared from the muscle in order to clean the separate muscle slips near its proximal attachment to the transverse processes of the cervical vertebrae. It was not necessary to reflect splenius capitis, but the muscle was cleaned in order to visualize the levator scapula properly. At this point the number of muscular slips could be identified and defined. Once the muscle was cleaned, the relevant measurements were taken (Figure 1).

With the aim of completely defining the origins of the levator scapulae muscles and their nerve innervation, the posterior approach was combined with an anterolateral approach. With the cadaver placed in the supine position and a support block used to extend the neck, the anterior triangle of the neck was dissected. A midline skin incision from chin to jugular notch and two transverse incisions; one along the inferior border of the mandible towards the mastoid process and second incision along the clavicle towards the acromion process. Superficial fascia, platysma and veins were removed. The sternocleidomastoid muscle was reflected towards its attachment to the mastoid process. The levator scapula was visible within the deep cervical fascia in the floor of the posterior triangle. Once this was done, the delicate process of defining the 
individual muscle slips and their attachment to the cervical vertebrae was done through blunt dissection and with the aid of an $\times 5$ dissection magnifying glass. The prevertebral layer of the deep cervical fascia surrounding the muscle was carefully removed. The anterior rami of the cervical spinal nerves $\mathrm{C} 3$ and $\mathrm{C} 4$ could now be identified. Twig-like branches to the anterior surface of the levator scapula were seen from these nerves. Intermuscular planes were used to define the levator scapula from other muscles in the floor of the posterior triangle. The fiber direction of the muscle and the union of the slips before inserting onto the scapula clearly defined the levator scapulae. Transition from muscle to tendon could be observed clearly, and each tendon could be followed to its attachment onto a vertebra. Measurements related to the origin of the levator scapula could now be made accurately and with ease.

Using a vernier dial caliper (R.S. Baty metric caliper $0-150 \mathrm{~mm} \pm 0.1 \mathrm{~mm}$ ) the following measurements were made:

A: The length of the insertion onto the upper medial border of the scapula.

B: Total length of the origin from the cervical vertebrae.

C: Length of the muscle from the highest origin to the superior insertion onto the medial border of the scapula.

All measurements were repeated three times and the averaged taken for accuracy. Muscle slips at origin were counted and recorded. Photographs for illustration purposes were taken with a Nikon D750 DSLR camera with a 24-120mm lens.

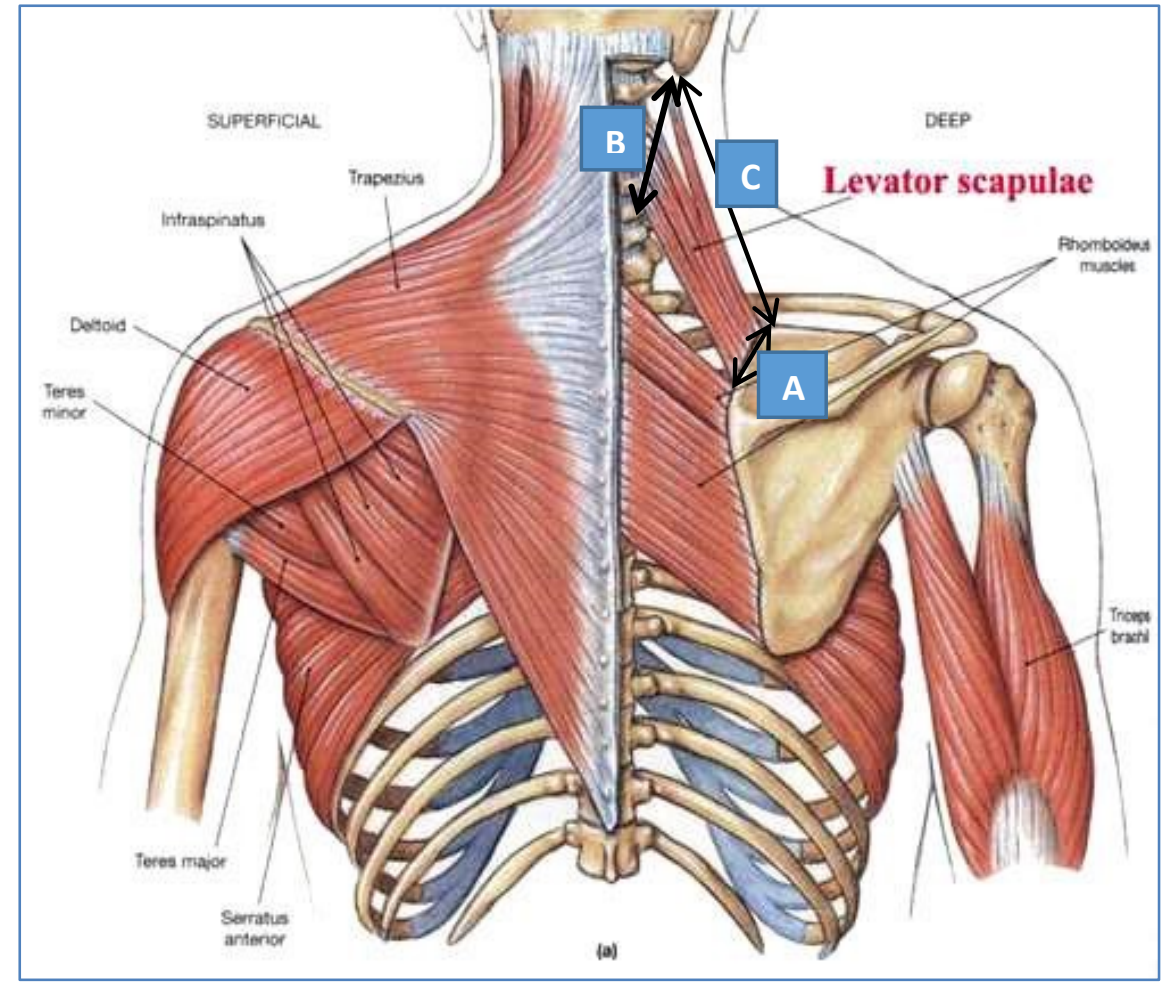

Figure 1: Arrow $A=$ insertion of the levator scapula; Arrow $B=$ origin of levator scapula; Arrow $C=$ length of the levator scapula (Martini et al., 2007). 


\section{RESULTS}

A total of 23 cadavers were used in this study. 21 cadavers were from the 2012 intake and 2 specimens were retained from the previous year. In total 46 levator scapulae muscles were used. All the sample values were measured to 2 decimal places. The average age of the cadavers in the sample was 82.87 years. The oldest cadaver in the sample was 100 years old and the youngest 61 years. Of the 23 specimens 15 were male. The leading cause of death in the male population was bronchopneumonia. The study population contained 8 female cadavers. The leading cause of death in the female population was also bronchopneumonia. Respiratory pathologies represented $78.3 \%$ of the deaths in the study group. Cardiac pathologies on the other hand only caused $8.7 \%$ of the fatalities.

\section{Number and length of Muscle Slips}

The number of muscle slips at the origin varied greatly. Table 1 summarizes the number of muscle slips of the levator scapulae which were investigated in this study. The average number of muscle slips was 4.11 with a standard deviation of 0.67 .

Figure 2 illustrates the distribution of the number of slips within the study population.

Three muscle slips were found in 7 of the 46 $(15.22 \%)$ levator scapulae. At the area of insertion on the medial border of the scapula, the mean length for levator scapulae with three muscle slips was $40.38 \mathrm{~mm}$ with a standard deviation of $3.42 \mathrm{~mm}$. The mean distance between superior origin and inferior origin on the transverse process of the cervical vertebrae was $37.58 \mathrm{~mm} \pm 5.60 \mathrm{~mm}$. The mean length of the 3-slip muscle was $118.60 \mathrm{~mm} \pm$ $16.41 \mathrm{~mm}$, measured from superior origin to insertion.

Of the levator scapulae investigated the majority were found to have four muscle slips. 28 of the 46 muscles had four slips, equating to $60.87 \%$ of the total. At the area of insertion on the medial border of the scapula the mean length was $46.54 \pm 5.37 \mathrm{~mm}$ standard deviation. The mean distance between superior origin and inferior origin on the transverse process of the cervical vertebrae was $48.98 \pm 8.93 \mathrm{~mm}$. The mean length of the levator scapulae with four slips was $105.40 \mathrm{~mm}$ $\pm 11.10 \mathrm{~mm}$, measured from superior origin to insertion.

The second most common findings were levator scapulae muscles with five slips. In total 10 were identified, corresponding to $21.74 \%$ of the total number investigated. The mean length of insertion on the scapula was found to be $45.34 \mathrm{~mm}$ with a standard deviation of $5.89 \mathrm{~mm}$. The mean distance between superior origin and inferior origin on the transverse process of the cervical vertebrae was $56.93 \mathrm{~mm} \pm 6.14 \mathrm{~mm}$. The mean length of the 5 slip muscle was $105.40 \mathrm{~mm} \pm$ $11.10 \mathrm{~mm}$, measured from superior origin to insertion.

Table 1: Variation in the number of muscle slips within the total sample; number and percentage.

\begin{tabular}{|l|l|l|}
\hline $\begin{array}{l}\text { Number of Muscle } \\
\text { Slips }\end{array}$ & $\begin{array}{l}\text { Number of levator } \\
\text { scapulae }\end{array}$ & $\begin{array}{l}\text { Percentage of Total } \\
(\%)\end{array}$ \\
\hline $\mathbf{3}$ & 7 & 15.22 \\
\hline $\mathbf{4}$ & 28 & 60.87 \\
\hline $\mathbf{5}$ & 10 & 21.74 \\
\hline $\mathbf{6}$ & 1 & 2.17 \\
\hline
\end{tabular}




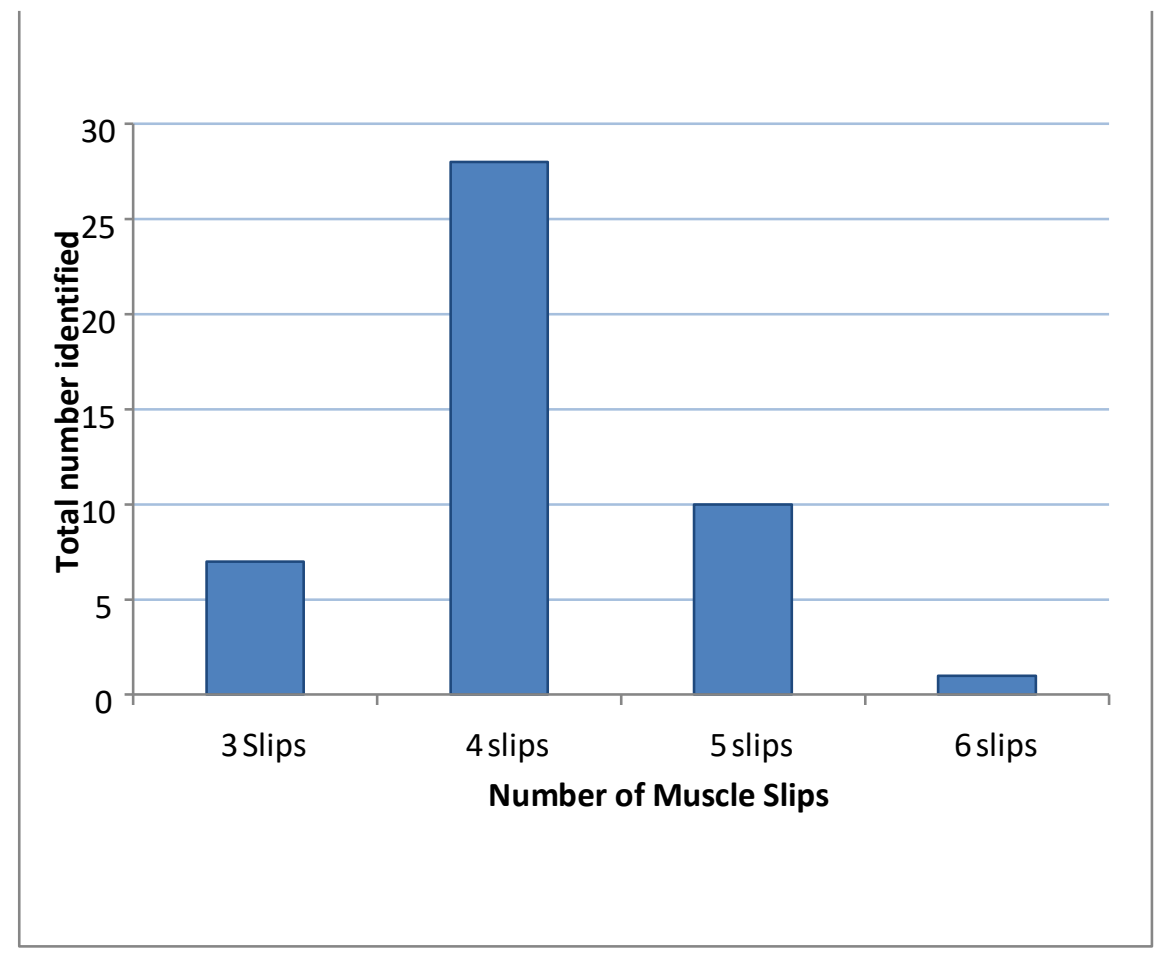

Figure 2: Bar chart illustrating the variation in the number of muscle slips.

Only one levator scapula muscle was found with six muscle slips; $2.17 \%$ of the total. This was found on the right side of cadaver $2010 / 21$. The muscle slips came from the transverse processes of the first 6 cervical vertebrae. The distance between the origin of the most superior and most inferior muscle attachments on the cervical vertebrae was $79.2 \mathrm{~mm}$. The area of insertion began at superior angle and continued to the root of the spine of scapula. The length of the insertion was measured at $41.3 \mathrm{~mm}$. The overall length of the muscle was measured at $131.4 \mathrm{~mm}$.

From this study sample, the mean length of the insertion on the medial border of the scapula was measured at $45.22 \mathrm{~mm}$ with \pm
$5.56 \mathrm{~mm}$ standard deviation. The mean distance between the superior and inferior origins of the muscle was $49.63 \mathrm{~mm}$ with a standard deviation of $\pm 9.46 \mathrm{~mm}$. The mean length of the muscle measured from the origin on the transverse process of $\mathrm{C} 1$ to the superior insertion on the scapula was $110.33 \mathrm{~mm}$ with a standard deviation of $\pm 11.61 \mathrm{~mm}$.

Table 2, Table 3 and Table 4 present statistical calculations to contrast the dimensions of the investigated levator scapulae, comparing the effect that the number of muscle slips had on the particular dimension of the muscle. 
Table 2: Statistical calculations used for length of insertion onto the medial border of scapula, comparing the number of muscle slips.

\begin{tabular}{|l|l|l|l|}
\hline Statistical Calculations & \multicolumn{4}{|l|}{ Length of insertion medial border of scapula (mm) } \\
\hline Number of Muscle Slips & $\mathbf{3}$ & $\mathbf{4}$ & $\mathbf{5}$ \\
\hline Total Number & 7.0 & 28.0 & 10.0 \\
\hline Mean (mm) & $40.4 \pm 3.4$ & $46.5 \pm 5.4$ & $45.3 \pm 5.9$ \\
\hline Minimum Value (mm) & 34.4 & 36.6 & 35.3 \\
\hline Maximum Value (mm) & 44.8 & 57.9 & 53.4 \\
\hline
\end{tabular}

Table 3: Length and number of muscle slips

\begin{tabular}{|l|l|l|l|}
\hline Statistical Calculations & \multicolumn{4}{|l|}{$\begin{array}{l}\text { Length from superior origin on transverse process of } \\
\text { C1 to insertion on medial border of the scapula (mm) }\end{array}$} \\
\hline Number of Muscle Slips & $\mathbf{3}$ & $\mathbf{4}$ & $\mathbf{5}$ \\
\hline Count & 7.0 & 28.0 & 10.0 \\
\hline Mean (mm) & $118.6 \pm 16.4$ & $109.3 \pm 8.9$ & $105.4 \pm 11.1$ \\
\hline Minimum Value (mm) & 92.7 & 98.7 & 94.5 \\
\hline Maximum Value (mm) & 138.9 & 135.5 & 128.7 \\
\hline
\end{tabular}

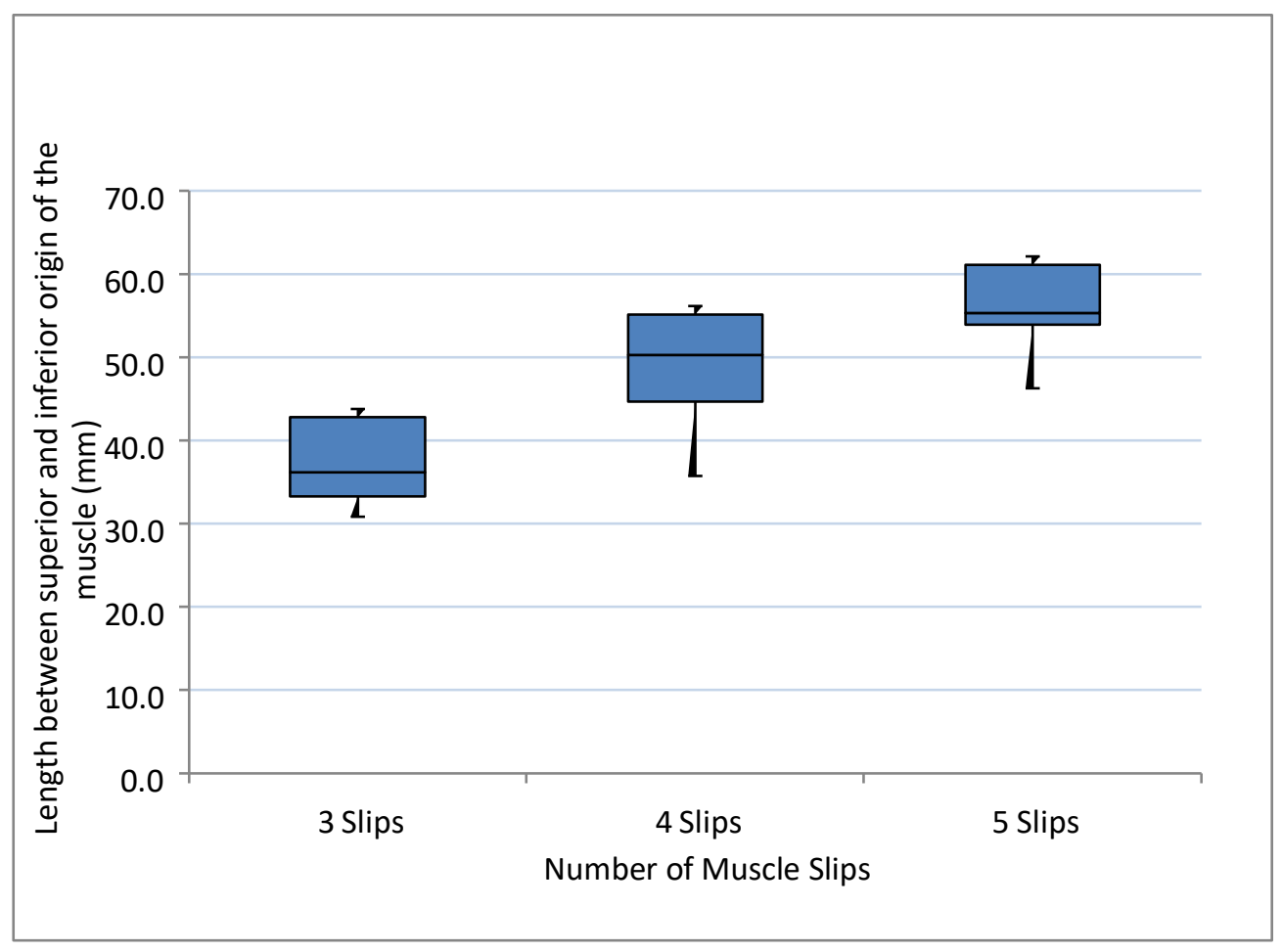

Figure 3: Box and whisker plot contrasting the distance between superior and inferior origins and the number of muscle slips. The values used to create the plot can be found in Table 4. 
Table 4: Statistical calculations used to compare the length between the superior and inferior origin on the transverse processes of the cervical vertebrae

\begin{tabular}{|l|l|l|l|}
\hline Statistical Calculations & \multicolumn{4}{|l|}{$\begin{array}{l}\text { Length Between Superior and Inferior origin on the transverse } \\
\text { processes of the cervical vertebrae }\end{array}$} \\
\hline Number of Muscle Slips & $\mathbf{3}$ & $\mathbf{4}$ & $\mathbf{5}$ \\
\hline Count & 7.0 & 28.0 & 10.0 \\
\hline Mean (mm) & $37.6 \pm 5.6$ & $49.0 \pm 6.3$ & $56.9 \pm 6.1$ \\
\hline Minimum Value (mm) & 30.8 & 35.7 & 46.3 \\
\hline Maximum Value (mm) & 44.0 & 56.8 & 68.3 \\
\hline
\end{tabular}

\section{Variation within Individual Cadavers}

The levator scapulae muscles on the two sides often varied. In 6 out of the 23 cadavers in the study, there was a difference in the number of muscle slips between the two sides. The most pronounced difference was in specimen 2011/14 which had 3 muscle slips on the left side and 5 muscle slips on the right side.

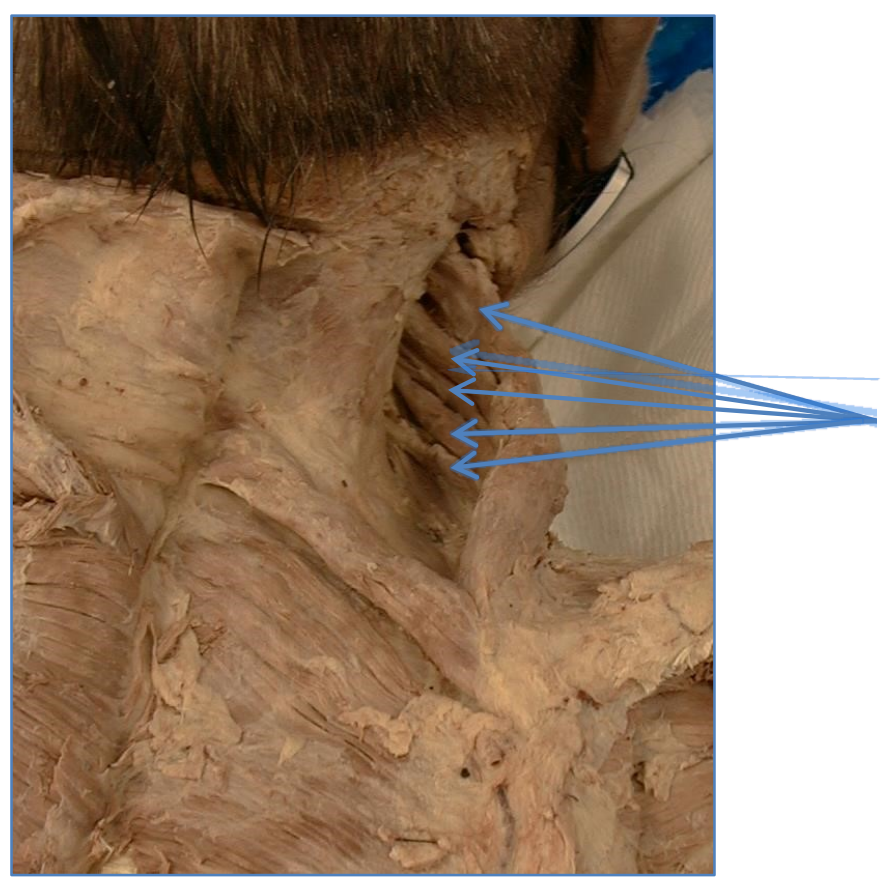

Figure 4: The posterior view shows the right levator scapula with 5 muscle slips -1 . Arrows indicate the 5 slips. The slips were attached to the transverse processes of cervical vertebrae C1- C5 


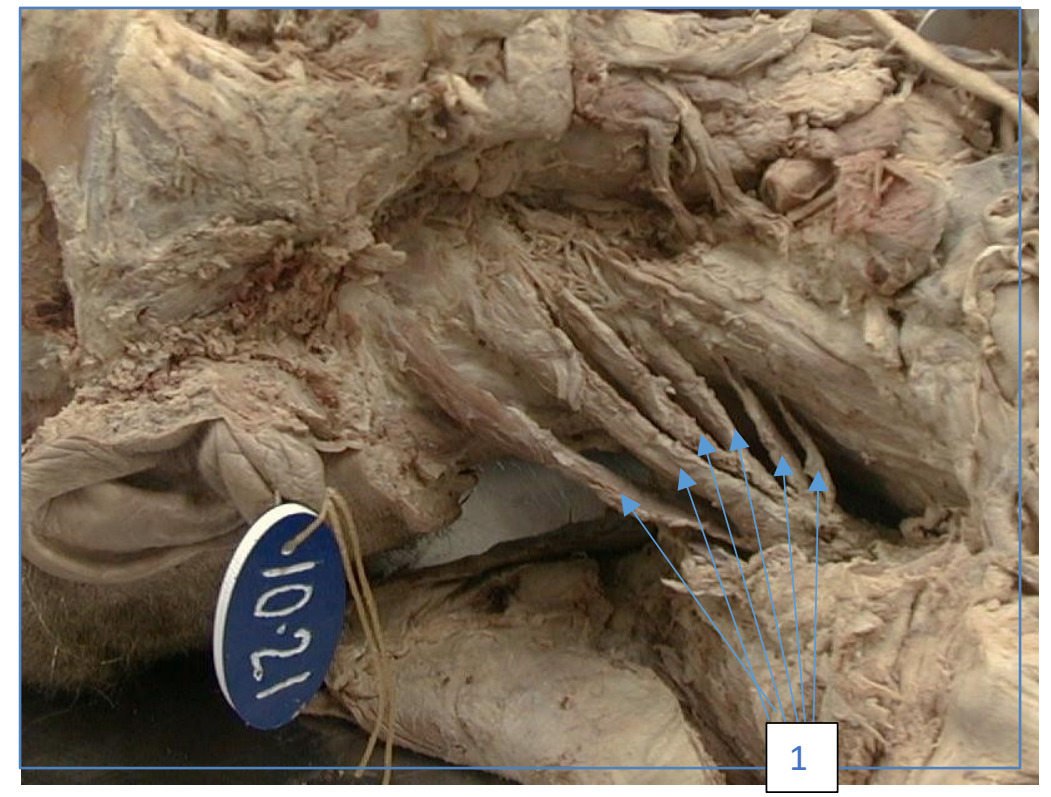

Figure 5: Anterolateral view of a levator scapula muscle with 6 slips. This was found on the right side of specimen $2010 / 21$. All slips unite near the insertion onto the scapula.

\section{DISCUSSION}

From our study population of 23 cadavers, the average age at time of death was 82.87 years. The male section of our study group had an average age of 83.87 years. This compares very well with the normal life expectancy of males in Northern Ireland which is predicted to be 76.4 years (NISRA, 2011). The average age for females sample was 81 years. This is higher than the expected age for females in Northern Ireland, which stands at 78.4 years (NISRA, 2011). Another interesting demographic in our study population was the high prevalence of respiratory causes of mortality, and in particular bronchopneumonia. Respiratory pathologies contributed to $78.3 \%$ of the total number of deaths in this group. Compare this to the total of mortalities as a consequence of respiratory disease in Northern Ireland which was reported in 2008 to be $14 \%$. Thought this figure excludes deaths due to lung cancer (NISRA, 2011). Also surprising was the low percentage of deaths caused by cardiovascular diseases which was only $8.7 \%$ of the total in our sample.

Unfortunately, the only information available to us, was the cause of death on the death certificate. There are no details regarding the general health of the person before death. As a consequence, it is hard to gauge if the muscle mass and dimensions could have been affected due to co-morbidities, immobility or age. Immobility is a recognized risk factor for bronchopneumonia, which was coincidentally the most common cause of death in our study population. The levator scapulae which were investigated in the study were found to have great variation. The variation in number of slips was much greater than anticipated. Of the 46 investigated levator scapulae just 7 levator scapulae were found to have 3 muscle slips. Each muscle with 3 slips had tendons arising from the transverse processes of cervical vertebrae $\mathrm{C} 1, \mathrm{C} 2$ and the posterior tubercle of the transverse process of C3. They all wrapped around the neck before inserting on the medial border of the scapula.

In total 28 levator scapulae were found with 4 muscle slips. This was the most common finding in our investigation, and this corresponds well with the textbook description in later publications. The muscle slips of the levator scapula originated on the transverse processes of the cervical vertebrae $\mathrm{C} 1-\mathrm{C} 4$. A 
total of 10 levator scapulae had 5 muscle slips. The slips originated from the first five cervical vertebrae.

In one case the right side the levator scapula had 6 muscle slips. The muscle slips were separated, and each slip originated from a different cervical vertebra. The first two muscle slips were very large and originated from the transverse process of $\mathrm{C} 1$ and $\mathrm{C} 2$ respectively. The following 4 muscle slips originated from the posterior tubercles of the transverse processes of the cervical vertebrae $\mathrm{C} 3$ to $\mathrm{C} 6$. After their origin on the cervical vertebrae the slips angled towards the scapula before uniting near the insertion onto the medial border of the scapula. While doing the literature review, no previous reports of a levator scapula with 6 distinct muscular slips of origin, could be found.

This investigation not only revealed a great variation of levator scapulae within the sample population, but also variations between levator scapulae within the same cadaver. In 6 cadavers out of the 23 there were different numbers of muscle slips on each side. In another specimen the right side had 5 muscle slips whereas the left had just four. The slips of this muscle had long tendons and thin muscle bellies.

In this study, great variation was found within the levator scapulae muscles. The average number of muscle slips found was 4.11 with a standard deviation of 0.67 . This compares favorably with other reports in literature. Mardones \& Torres found the average number of muscle slips to be 3.95 with a standard deviation of 0.85 . Their study contained 11 cadavers and was prompted by the recent applications and surgical techniques involving the levator scapula (Mardones \& Torres, 2006). Another study also gained similar results, finding the average number of muscle slips originating from the cervical vertebrae to be 4.05 (Macbeth \& Martin, 1953). The standard deviation also indicates that variation in the number of slips was found in other studies.

An anatomical study carried out in Japan observing and documenting the differences in musculature in a population of 50 cadavers, found $66.6 \%$ of levator scapulae to have 4 muscle slips. 26.6\% had 3 muscle slips and just $3.3 \%$ were found to have 5 muscle slips (Mori, 1964). Interestingly, this contradicts the findings of this study. Although in our study the most common finding was also 4 muscle slips $(60.87 \%)$, the next most frequent finding was 5 muscle slips (21.74\%). 3 slips only accounted for $15.22 \%$ of the total levator scapulae investigated. In our study the prevalence of 5 muscle slips are therefore much higher. However, the prevalence of 3 slips are much lower. None of the above-mentioned studies reported a levator scapula with 6 muscle slips.

For the tables comparing the dimensions of levator scapula, I omitted the specimen with 6 slips as this was insufficient for statistical analysis. Instead muscles with 3, 4 and 5 slips were compared. The area of insertion of the levator scapula on the medial border of the scapula was represented by the length between the superior and inferior points of insertion. The mean length of insertion was $45.22 \mathrm{~mm}$ and the standard deviation was $5.56 \mathrm{~mm}$. Standard deviation was used as an indicator of the degree of spread of the data around the mean. The standard deviation of the entire population was quite small showing that the length of insertion in the study population was quite similar. The median length of insertion for 3 slips $(40.1 \mathrm{~mm})$ was smaller than the insertion of muscles with 4 and 5 slips. The quartile ranges however, showed little difference, especially Q1. Therefore, there was not significant differences in the length of the insertion between 3, 4 and 5 muscle slips.

The length of each muscle was estimated by measuring the distance between the most superior attachments on the transverse process of $\mathrm{C} 1$, to the most superior point of insertion on the medial border of scapula. The mean length of the levator scapula was $110.33 \mathrm{~mm} \pm 11.61 \mathrm{~mm}$. The standard deviation was highest for this measurement, meaning the spread of results was greater. It was found that the length of the muscle increased as the number of muscle slips 
decreased. The mean length of the muscle was $118.6 \mathrm{~mm}$ for muscles with 3 slips, $109.3 \mathrm{~mm}$ for muscles with 4 slips and $105.4 \mathrm{~mm}$ for muscles with 5 slips. It must be noted that the standard deviation of the muscles with 4 slips was smallest even though there were 28 levator scapulae with 4 slips recorded, many more than in the other groups. If this study was expanded to include greater numbers and the same conclusions were made, this finding would be very useful in clinical applications.

The mean distance between the superior and inferior origins of the muscle on the transverse processes of the cervical vertebrae was $49.63 \mathrm{~mm} \pm 9.46 \mathrm{~mm}$. The graph shows a gradual increase in the distance between the superior and inferior origin as the number of muscle slips increases. The mean values also demonstrate this progression. It is logical that the distance between the superior and inferior bellies of the muscle increases as the number of slips increases; as the slips tend to rise from the transverse processes of adjacent cervical vertebra. In the cadaver with 6 slips the distance was $79.2 \mathrm{~mm}$, which demonstrates this progression the best. The relatively wide dispersion of muscle dimensions and muscle slips obtained from the levator scapulae in this study show that the muscle is far from consistent in nature. This degree of variation should be of interest to clinicians concerned in this area.

Following the results obtained from this small study, we believe there is great potential for further research to be carried out on the levator scapula muscle. Firstly, a larger sample would be beneficial in helping to generate more reliable data. This could be achieved by continuing the research and collating the results or indeed combining the results with data from clinical imaging techniques such as MRI, CT or ultrasound. In the introduction, we highlighted the clinical relevance of the levator scapula and its importance with neck and shoulder pain. It has in the past been postulated that anatomical anomalies could play a role in the development of shoulder pain. When considering the delicate balance required, maintaining posture, alignment of the cervical vertebrae, and complex movements of the shoulder, muscular variations could upset this balance and lead to soft tissue pain symptoms. Therefore, it would be of great interest to investigate the levator scapulae in patients who suffer from myofascial shoulder pain with an investigating anatomical / clinical study. Visualisation of the levator scapula has been attempted by using ultrasound in a small sample group. Out of the twenty volunteers recruited, excellent visualisation of the levator scapulae was achieved in 11 out of 20 cases (Saranteas et al., 2010). Although ultrasound is a low cost and simple method of investigation, the reliability and accuracy of the results could not rival that of an MRI scan. Data from MRI scans would be ideal for their accuracy, although they are not done routinely for patients with neck and shoulder pain. This is due to the fact that an MRI scan is unlikely to alter the treatment plan of these patients and is very expensive. An anatomical / clinical study definitely has potential, and calls have been made for such a study (Menachem et al., 1993). Another possible extension to the project would be to investigate whether there is a higher incidence of complications during a brachial plexus block in patients who have anatomical variations in the posterior triangle, compared to patients without anatomical variations.

Again, this would be difficult as it also relies on accurate visualization of the levator scapulae by using scans. The same principle could be applied to surgical complications, such as radical neck dissection for head and neck cancers and anatomical anomalies. This would have more promise, as most patients with head and neck cancers will have several investigations to visualize the area of concern before surgery.

In conclusion levator scapulae shows variations in the number of muscle slips at the origin from the cervical vertebrae. In recent Anatomy textbooks, 4 muscle slips are described for the levator scapulae. However, in our study we report 3 to 6 muscle slips in our study group. In order to improve the reliability of the results of this study a greater number of specimens should be used, either through further dissection or inclusion of results from imaging 
techniques. Nonetheless, the findings of the research should still be of great interest to clinicians associated with this area of the body. Future directions could see the study expanded to include patients with neck and shoulder pain. By doing this the speculated link between muscle variation and clinical outcomes such as myofascial pain syndrome could be investigated further. The potential applications and expansions of this study are exciting and may begin to uncover the unexplained mechanisms behind myofascial pain syndrome.

\section{REFERENCES}

1. Loukas M, Louis RG Jr, Merbs W. 2006. A case of atypical insertion of the levator scapulae. Folia morphologica, vol. 65, no. 3, pp. 232-235.

2. Macbeth RA, Martin CP. 1953. A note on the levator scapulae muscle in man. The Anatomical Record, vol. 115, no. 4, pp. 691-696.

3. Mardones VF, Torres RA. 2006. Levator Scapulae Muscle. International Journal of Morphology. 24: 251-258.

4. Martini F, Bartholomew EF, Ober WC. 2007. Essentials of anatomy \& physiology / Frederic $H$. Martini, Edwin F. Bartholomew; 4th Edition., San Francisco; London: Pearson \& Benjamin Cummings Picture adapted from pp. 266.

5. Menachem A, Kaplan O, Dekel S. 1993. Levator scapulae syndrome: an anatomicclinical study. Bulletin (Hospital for Joint Diseases (New York, N.Y.)), vol. 53, no. 1, pp. 21-24.

6. Mori M. 1964. "Statistics on the Musculature of the Japanese", Okajimas folia anatomica Japonica, vol. 40, pp. 195-300.

7. NISRA, Northern Ireland Statistics and Research Agency. 2011. NI Health \& Social Care Inequalities Monitoring System [Homepage of Department of Health, Social Services and Public Safety Northern Ireland], Online. Website updated- 20/02/11, Accessed 24/04/12 Available

URL: http://www.dhsspsni.gov.uk/hscims life expectancy decomposition 2011.pdf

8. Rubinstein D, Escott EJ, Hendrick LL. 1999. The prevalence and CT appearance of the levator claviculae muscle: a normal variant not to be mistaken for an abnormality. AJNR.American journal of neuroradiology, vol. 20, no. 4, pp. 583-586.

9. Saranteas T, Paraskeuopoulos T, Anagnostopoulou S, Kanellopoulos I, Mastoris M, Kostopanagiotou G. 2010. Ultrasound anatomy of the cervical paravertebral space: a preliminary study, Surgical and radiologic anatomy: SRA, vol. 32, no. 6, pp. 617622.

10. Standring S. 2005. Gray's AnatomyThe Anatomical Basis of Clinical Practice, 39th Edition, Elsevier Churchill Livingstone, Philadelphia.

11. Wood J. 1867. On Human Muscular Variations and their Relation to Comparative Anatomy. Journal of anatomy and physiology, vol. 1, no. 1, pp. 44-59. 